\title{
MODELING AND SIMULATION OF PRESSURE, TEMPERATURE AND CONCENTRATION FOR THERMAL EXPLOSIONS
}

\author{
OLIMPIA BUNTAa , MIHAELA-LIGIA UNGUREŞAN ${ }^{\mathrm{b}, *}$, \\ VLAD MUREŞAN ${ }^{\mathrm{c}}$, OVIDIU STAN ${ }^{\mathrm{c}}$
}

\begin{abstract}
In this paper is presented a simple possible model which can explain the thermal explosion problem, the existence of an induction period and a sudden rapid temperature rise. As state variables used for modeling are: the pressure, the temperature and the concentration. The time evolutions of these state parameters are analogically modeled using ordinary differential equations. The numerical simulations of the obtained model are made in Matlab/Simulink ${ }^{\mathrm{TM}}$. The validation of the model is realized by comparison between experimental data and simulation results, presenting a good accuracy.
\end{abstract}

Keywords: thermal explosion, analogical modeling, numerical simulation, state Parameters

\section{INTRODUCTION}

Thermal explosions are events of high complexity which typically involve several processes from the fields of chemistry and physics, such as temperature rises [1], expansion, phase transition, chemical processes and reactions, and finally momentum transfer [2]. The analysis of an explosion phenomena may involve the description of the physical and chemical initial states of the material, its rate of heating, rate of decomposition kinetics [3], changes in the chemical and mechanical explosive properties, rate of burning, transfer rate of explosive energy into thermal and mechanical energy (hydrodynamics), and, finally, amount of damage (violence) $[4,5]$.

\footnotetext{
a Iuliu Hatieganu University of Medicine and Pharmacy, Orthodontics Department, 31 Avram lancu st., 400117, Cluj-Napoca, Romania

b Technical University of Cluj-Napoca, Physical and Chemistry Department, 103-105 Muncii st., 400641, Cluj-Napoca, Romania

c Technical University of Cluj-Napoca, Department of Automation, 15 C-tin Daicoviciu st., 400020, Cluj-Napoca, Romania

*Corresponding author: mihaela.unguresan@chem.utcluj.ro
} 
In a thermal explosive reaction, the rate of reaction rapidly varies with temperature and the moment of explosion takes place at some finite temperature $[6,7]$.

The value of the critical temperature, below which the thermal explosion of a chemical cannot occur, is indispensable to prevent such a chemical from exploding. To determine the critical temperature, it has so far been necessary to measure the value in explosion experiments [8].

The problem of the critical regimes evaluation, perceived as separating regimes of the explosive regions and nonexplosive ways of the chemical reactions, is the most important mathematical aspect within the thermal explosion theory. Many authors, such as N. N. Semenov [9], D. A. FrankKamenetsky [10], O. M. Todes and P. V. Melent'ev [11], A. G. Merzhanov and F. I. Dubovitsky [12], B. Grey [13], have investigated the critical phenomena of the thermal explosion theory.

A solution for modelling the real phenomena, including, using fractional order differential equations (which can describe the mathematical models of the fractional order systems), is presented.[14] The fractional order models have the main advantage of generating a wider spectrum of possible approximations for the behaviour of the approached phenomena. This aspect implies the possibility to obtain more accurate models than using integer order models. However, the fractional order models have to main disadvantages: firstly, these types of models present more parameters than the integer order ones, fact that make the identification of the approached phenomena dynamics more difficult (the applied identification methods are based, mainly, on iterative algorithms); secondly, the numerical simulation of fractional order models involve complex problems (the necessity of their approximation using integer order models occurs, using, for example, the Oustoloup filter, but the resulted approximation have, in general, significantly high orders, their simulation becoming laborious), aspect which make difficult their usage in practice.

Some interesting dependency functions between the parameters of the phenomenon of recombination of Oxygen and nitrogen atoms on quartz are presented in [15]. However, the proposed functions, describing static mathematical dependences, cannot be used for the accurate modelling of the explosion dynamics. In [16], a predictive modelled is used for the lower flammability limits of $\mathrm{H}_{2} / \mathrm{O}_{2} / \mathrm{CO}_{2}$ mixture in order to prevent the explosion.

Examples of analytically determined mathematical models based on usage of ordinary differential equations and partial differential equations.[17] However, these models are valid for particular types of thermal explosions and, in the case when experimental data are available, their usage questionable. 
In this paper, an original and general method for the modelling and simulation of the thermal explosions is presented. The numerical simulation method based on Taylor series generates high accuracy and it can be efficiently applied both if the considered thermal explosion is modelled as lumped parameter process or as distributed parameter model. In the case of modelling the explosions as lumped parameter models, the ordinary differential equations are use and in the case of modelling the explosions as distributed parameter models, the partial differential equations are used. The proposed method is efficient in the case when experimental data must be used in the modelling procedure. To prove the proposed method efficiency, the case of thermal explosion of oxyhydrogen gas initiated by the wall of a quartz reactor [18] is considered.

\section{RESULTS AND DISCUSSION}

\section{Mathematical modeling}

It is a known fact that the usual linear processes can be expressed through the following analytical model:

$$
\begin{aligned}
& \dot{\mathbf{x}}=\mathbf{A x}+\mathbf{B u} \\
& \mathbf{y}=\mathbf{C x}+\mathbf{D u}
\end{aligned}
$$

where: $\mathbf{u}=\mathbf{u}(\mathrm{t}), \mathbf{x}=\mathbf{x}(\mathrm{t})$ and $\mathbf{y}=\mathbf{y}(\mathrm{t})$ represent the input, the state and the output vectors, and (A), (B), (C), and (D) correspond to the state, the inputstate, the state-output, and the input-output matrix. The matrices are constant if the process is time invariant and variable if the process varies in time. The initial conditions (IC), for $t=t_{0}$, respectively $\mathbf{x}_{I C}=\mathbf{x}\left(t_{0}\right)$ are known. In the hypothesis in which the known $\mathbf{u}=\mathbf{u}(\mathrm{t})$ input vector presents $a$ continuous evolution in relation to time, the solution in its vectorial form (1) of the ordinary differential equation (ode), respects the conditions of continuity of the Cauchy sense.

Taking into consideration a limited number of successive derivatives which consider the time variable for (1), respectively:

$$
\begin{gathered}
\dot{\mathbf{x}}=\mathbf{A} \mathbf{x}+\mathbf{B u} \\
\ddot{\mathbf{x}}=\dot{\mathbf{A}} \mathbf{x}+\mathbf{A} \dot{\mathbf{x}}+\dot{\mathbf{B}} \mathbf{u}+\mathbf{B} \dot{\mathbf{u}} \\
\dddot{\mathbf{x}}=\ddot{\mathbf{A}} \mathbf{x}+2 \dot{\mathbf{A}} \dot{\mathbf{x}}+\mathbf{A} \ddot{\mathbf{x}}+\ddot{\mathbf{B}} \mathbf{u}+2 \dot{\mathbf{B}} \dot{\mathbf{u}}+\mathbf{B} \ddot{\mathbf{u}} \\
\dddot{\mathbf{x}}=\dddot{\mathbf{A}} \mathbf{x}+3 \ddot{\mathbf{A}} \dot{\mathbf{x}}+3 \dot{\mathbf{A}} \ddot{\mathbf{x}}+\mathbf{A} \dddot{\mathbf{x}}+\dddot{\mathbf{B}} \mathbf{u}+3 \ddot{\mathbf{B}} \dot{\mathbf{u}}+3 \dot{\mathbf{B}} \ddot{\mathbf{u}}+\mathbf{B} \ddot{\mathbf{u}}
\end{gathered}
$$


It can be observed that $(\dot{\mathbf{x}})$ in (4) derives from (3), ( $\ddot{\mathbf{x}})$ in (5) derives from (4), and finally ( $\dddot{\mathbf{x}}$ ) in (6) derives from (5), and so on, which simplifies the operation of progressive derivatives which regard the time variable of the state vector $(\mathbf{x})$.

The present paper proposes the method of the complete Taylor series for the numerical estimation of the $\left(\mathbf{x}_{\mathrm{k}}\right)$ vector:

$$
\mathbf{x}_{\mathbf{k}} \cong \mathbf{x}_{\mathbf{k}-1}+\sum_{\mathrm{m}=1}^{\omega} \frac{\Delta \mathrm{t}^{\mathrm{m}}}{\mathrm{m} !} \mathbf{x}_{\mathbf{k}-1}
$$

The right side of the equation is considered at the $t_{k-1}=(k-1) \cdot \Delta t$ moment, where (k-1) stands for the regressive sequence, and the sufficiently undersized $(\Delta t)$, stands for the integration step. Therefore, the estimated solution at the $t_{k}=k \cdot \Delta t$ moment, where $(k)$ represents the current sequence, corresponds to:

$$
\mathrm{x}_{\mathrm{k}} \cong \mathrm{x}_{\mathrm{k}-1}+\sum_{\mathrm{m}=1}^{\omega} \frac{\Delta \mathrm{t}^{\mathrm{m}}}{\mathrm{m} !}\left(\mathrm{A} \cdot \mathrm{x}_{(\mathrm{k}-1)}^{(\mathrm{m}-1)}+\mathrm{B} \cdot \mathrm{u}_{(\mathrm{k}-1)}^{(\mathrm{m}-1)}\right)
$$

where $(\omega)$ marks the maximum derivatives number with respect to the time variable, which limits the truncated evolution of the Taylor series, for the situation of the minimal $\omega \geq 2$ condition. Obviously: $\mathbf{u}_{k-1}=\mathbf{u}\left(\mathrm{t}_{\mathrm{k}-1}\right), \mathbf{x}_{\mathrm{k}-1}=\mathbf{x}\left(\mathrm{t}_{\mathrm{k}-1}\right)$, $\mathbf{x}_{\mathrm{k}}=\mathbf{x}\left(\mathrm{t}_{\mathrm{k}}\right)$, and $\mathrm{u}_{(\mathrm{k}-1)}^{(\mathrm{m}-1)}=\left[\frac{\mathrm{d}^{\mathrm{m}-1}}{\mathrm{dt}^{\mathrm{m}-1}}(\mathrm{u})\right]_{\mathrm{k}-1}, \mathrm{x}_{(\mathrm{k}-1)}^{(\mathrm{m}-1)}=\left[\frac{\mathrm{d}^{\mathrm{m}-1}}{\mathrm{dt}^{\mathrm{m}-1}}(\mathrm{x})\right]_{\mathrm{k}-1}$ respectively with the remark that for $m=1$, respectively $(m-1)=(0)$, correspond (0) (0) $\mathbf{u}_{\mathbf{k}-1}=\mathbf{u}_{\mathbf{k}-1}$ and $\mathbf{x}_{\mathbf{k}-1}=\mathbf{x}_{\mathbf{k}-1}$.

In order to ensure the starting of the calculation, at the initial $t_{0}=t_{k-1}$, respectively $\mathrm{k}=1$ moments, the investigated initial conditions are expressed by using the $\left(\mathbf{x}_{0}\right)$ state vector, to which the $\left(\mathbf{u}_{0}\right)$ input vector is associated.

The vector $\left(\mathbf{x}_{\mathrm{k}}\right)$ approximated at the $t_{k}=k \cdot \Delta t$ moment, corresponds to:

$$
\begin{aligned}
& \mathbf{x}_{\mathrm{k}} \cong \mathbf{x}_{\mathrm{k}-1}+\frac{\Delta \mathrm{t}}{1 !}(\mathbf{A} \mathbf{x}+\mathbf{B} \mathbf{u})_{\mathrm{k}-1}+\frac{\Delta \mathrm{t}^{2}}{2 !}(\dot{\mathbf{A}} \mathbf{x}+\mathbf{A} \dot{\mathbf{x}}+\dot{\mathbf{B}} \mathbf{u}+\mathbf{B} \dot{\mathbf{u}})_{\mathrm{k}-1}+ \\
&+\frac{\Delta \mathrm{t}^{3}}{3 !}(\ddot{\mathbf{A}} \mathbf{x}+2 \dot{\mathbf{A}} \dot{\mathbf{x}}+\mathbf{A} \ddot{\mathbf{x}}+\ddot{\mathbf{B}} \mathbf{u}+2 \dot{\mathbf{B}} \dot{\mathbf{u}}+\mathbf{B} \ddot{\mathbf{u}})_{\mathrm{k}-1}+ \\
&+\frac{\Delta \mathrm{t}^{4}}{4 !}(\dddot{\mathrm{A}} \mathbf{x}+3 \ddot{\mathbf{A}} \dot{\mathbf{x}}+3 \dot{\mathbf{A}} \ddot{\mathbf{x}}+\mathbf{A} \dddot{\mathbf{x}}+\ddot{\mathbf{B}} \mathbf{u}+3 \ddot{\mathbf{B}} \dot{\mathbf{u}}+3 \dot{\mathbf{B}} \ddot{\mathbf{u}}+\mathbf{B} \ddot{\mathbf{u}})_{\mathrm{k}-1}+\ldots
\end{aligned}
$$

The ()$_{k-1}$ notation highlights the fact that the whole expression between the parenthesis is considered at the $(k-1)$ sequence and at the $t_{k-1}=(k-1) \cdot \Delta t$ moment. 
For the considered process the time evolution of the three state parameters, respectively the pressure (Figure 1), the temperature (Figure 2) and the concentration (Figure 3) are approximated by the periodical exponential functions in the form:

$$
y_{\text {AN }}(t)=\left(1-\frac{T_{1}}{T_{1}-T_{2}} \varepsilon^{-t / T_{1}}-\frac{T_{2}}{T_{2}-T_{1}} \varepsilon^{-t / T_{2}}\right) \cdot K \cdot u
$$

for the increasing evolutions in Figure 1 and 2, respectively in the form:

$$
y_{A N}(t)=\left(\frac{T_{1}}{T_{1}-T_{2}} \varepsilon^{-t / T_{1}}+\frac{T_{2}}{T_{2}-T_{1}} \varepsilon^{-t / T_{2}}\right) \cdot K \cdot u
$$

for the decreasing evolution in Figure 3 . The input signal $u_{0}=$ constant is considered to be the one to induce the explosion, and $(\mathrm{K})$ represents a proportionality coefficient.

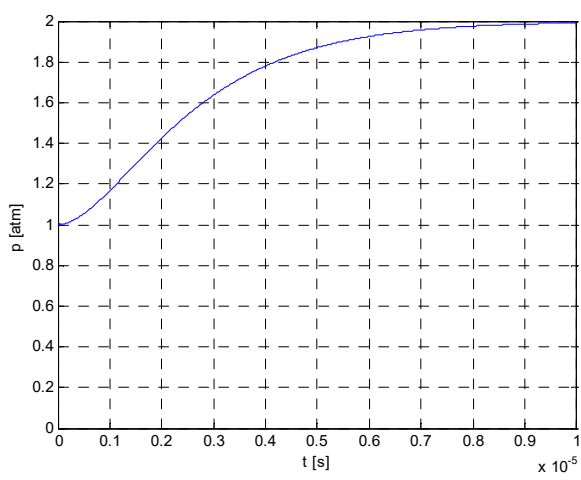

Figure 1. The evolution of pressure

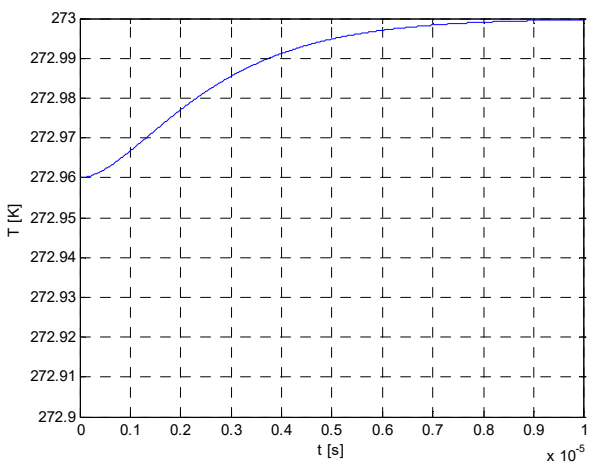

Figure 2. The evolution of temperature 


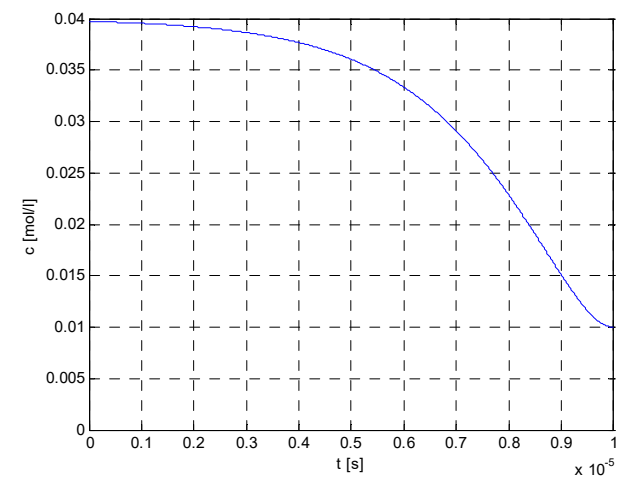

Figure 3. The evolution of concentration

The two-time constants $\left(T_{1}\right)$ and $\left(T_{2}\right)$ are specific for each of the state parameters, respectively:

$$
T_{2}=\lambda \cdot T_{1}
$$

and the final $t_{f}=10^{-5}$ time is approximated by:

$$
t_{f}=\mu \cdot\left(T_{1}+T_{2}\right)=\mu \cdot(1+\lambda) \cdot T_{1}=\mu \cdot\left(1+\frac{1}{\lambda}\right) \cdot T_{2}
$$

where $\mu=5$ and $\lambda=2$ are considered.

The point of inflexion of these curves results at the time:

$$
T_{i}=\frac{T_{1} T_{2}}{T_{2}-T_{1}} \cdot \ln \left(\frac{T_{1}}{T_{2}}\right)=\mu \cdot \frac{\lambda+1}{\lambda-1} \cdot \ln \lambda \cdot t_{f}
$$

so that the decrease of $\lambda>1$ restricts the progressive decrease of the inflexion moment $\left(\mathrm{t}_{\mathrm{i}}\right)$.

The analogical model associated to functions (1) and (2) can be expressed using the ordinary differential equation:

$$
A_{0} \cdot y+a_{1} \frac{d y}{d t}+a_{2} \frac{d^{2} y}{d t^{2}}=a_{0} y_{0}+a_{1} y_{1}+a_{2} y_{2}=K \cdot u_{0}
$$

where $a_{0}=1 ; a_{1}=T_{1}+T_{2}$ and $a_{2}=T_{1} \cdot T_{2}$.

The initial conditions considered at the $\mathrm{t}=\mathrm{t}_{0}=0$ moment are:

- for Figure 1: $y_{01 C}=1 ; y_{11 C}=0 ; u_{0}=1$;

- for Figure 2: $y_{01 c}=272.96 ; y_{11 C}=0 ; u_{0}=0.04$;

- for Figure 3: $\mathrm{y}_{01 \mathrm{C}}=0 ; \mathrm{y}_{11 \mathrm{C}}=0 ; \mathrm{u}_{0}=0.03$.

This model stood at the base of the software elaborated in order to obtain the numerical simulation. 
MODELING AND SIMULATION OF PRESSURE, TEMPERATURE AND CONCENTRATION FOR ..

\section{The numerical simulation}

Using the presented method, for the present application results the following algorithm:

$$
\begin{gathered}
y_{0}=y_{01} \\
y_{1}=y_{11}
\end{gathered}
$$

where $\mathrm{K}=1, \mathrm{u}_{0}=1 ; \mathrm{u}_{1}=0 ; \mathrm{u}_{2}=0$ and $\mathrm{u}_{3}=0$.

The above iterations continue with two Taylor Series, which will replace (16) and (17), respectively:

$$
y_{0 K}=y_{0}+\frac{\Delta t}{1 !} y_{1}+\frac{\Delta t^{2}}{2 !} y_{2}+\frac{\Delta t^{3}}{3 !} y_{3}+\frac{\Delta t^{4}}{4 !} y_{4}+\frac{\Delta t^{5}}{5 !} y_{5}
$$

and

$$
y_{1 K}=y_{1}+\frac{\Delta t}{1 !} y_{2}+\frac{\Delta t^{2}}{2 !} y_{3}+\frac{\Delta t^{3}}{3 !} y_{4}+\frac{\Delta t^{4}}{4 !} y_{5}
$$

where $\Delta t=t_{f} / 100$ is the integration step.

The indicator for this numerical integration performance is expressed by "the relative error cumulated in percentages" [19], denoted with:

$$
\text { Crep } y_{0}=100 \cdot \frac{\sum_{K=0}^{K_{f}} \Delta y_{0 K}}{\sum_{K=0}^{K_{f}} y_{A N, K}}
$$

where $\mathrm{K}_{0}=0$, respectively $\mathrm{K}_{\mathrm{f}}=\mathrm{t}_{\mathrm{f}} / \Delta \mathrm{t}$ are the initial and final sequences of calculation. The sums $\left(\sum_{\mathrm{K}=0}^{\mathrm{K}_{\mathrm{f}}} \mathrm{y}_{\mathrm{AN}, \mathrm{K}}\right)$ result from (1) and (2) and: 


$$
\sum_{K=0}^{K_{f}} \Delta y_{0 K}=\left|\sum_{K=0}^{K_{f}}\left(y_{0 K}-y_{0 A N, K}\right)\right|
$$

The indicator (15) is established at each calculus iteration, and the $\left(t=t_{f}\right)$ final values are in the range of the $\left(10^{-4}\right) \%$ order, which proves the remarkable accuracy of this numerical integration method.

The above algorithm was implemented in Matlab ${ }^{\text {TM }}$ software. The simulation results are presented in Table 1 , successively grouped on Figures 1,2 and 3 for the time interval $t_{0}=0$, up to $t_{f}=10^{-5}$, with the extraction step of the results of $\Delta t=10 \cdot \Delta t=10^{-6}$.

Table 1. Simulation results of thermal explosion state variables

\begin{tabular}{|l|c|c|c|c|c|c|}
\hline t/s & $10^{-7}$ & $1.1 \cdot 10^{-6}$ & $2.1 \cdot 10^{-6}$ & $3 \cdot 10^{-6}$ & $4 \cdot 10^{-6}$ & $5 \cdot 10^{-6}$ \\
\hline Pressure/(atm) & 1.0052 & 1.3155 & 1.628 & 1.800 & 1.903 & 1.953 \\
\hline Temperature (K) & 272.960 & 272.978 & 272.985 & 272.99 & 272.996 & 272.998 \\
2 & 9 & 2 & 2 & 1 & 2 \\
\hline $\begin{array}{l}\text { Concentration } \\
\text { (mol/l) }\end{array}$ & 0.03984 & 0.03053 & 0.02113 & $\begin{array}{c}0.0159 \\
9\end{array}$ & 0.01291 & 0.01139 \\
\hline Crep (\%) & $10^{-4}$ & $10^{-4}$ & $10^{-4}$ & $10^{-4}$ & $10^{-4}$ & $10^{-4}$ \\
\hline
\end{tabular}

\begin{tabular}{|l|c|c|c|c|c|}
\hline t/s & $6 \cdot 10^{-6}$ & $7 \cdot 10^{-6}$ & $8.1 \cdot 10^{-6}$ & $9.1 \cdot 10^{-6}$ & $10^{-5}$ \\
\hline Pressure/(atm) & 1.979 & 1.990 & 1.995 & 1.9978 & 1.9989 \\
\hline Temperature (K) & 272.9992 & 272.9996 & 272.9998 & 272.9999 & 273 \\
\hline $\begin{array}{l}\text { Concentration } \\
\text { (mol/l) }\end{array}$ & 0.010615 & 0.0103 & 0.010137 & 0.010065 & 0.0100307 \\
\hline Crep (\%) & $10^{-4}$ & $10^{-4}$ & $10^{-4}$ & $10^{-4}$ & $10^{-4}$ \\
\hline
\end{tabular}

The used parameters were:

- the integration step $\Delta t=10^{-9}$;

- the calculus coefficients for the time constants: $\mu=5 ; \lambda=2$;

- the time constants: $T_{1}=6.67 \cdot 10^{-7} ; T_{2}=1.33 \cdot 10^{-6}$;

- the coefficients of the equation (15): $a_{0}=1 ; a=2 \cdot 10^{-6} ; a_{2}=8.88 \cdot 10^{-7}$;

- crep $y_{0}$ is of the order $10^{-4}$, which proves the remarkable performance of the numerical integration. 
The inflexion moment $\left(t_{i}\right)$ in (14) can be changed in wide limits. For this particular study and using the corresponding software, it was obtained $t_{i}$ $=9.24 \cdot 10^{7}$, for a number of 1000 integration steps.

Example: The thermal explosion of oxyhydrogen gas initiated by the wall of a quartz reactor

The experimental data obtained during a thermal explosion of oxyhydrogen gas initiated by the wall of a quartz reactor are given. The experimental data are referring to the pressure variation and to the temperature variation during the explosion. The data associated to the pressure variation (the derivative of pressure in relation to time) are given in Table 2.

Table 2. The pressure variation

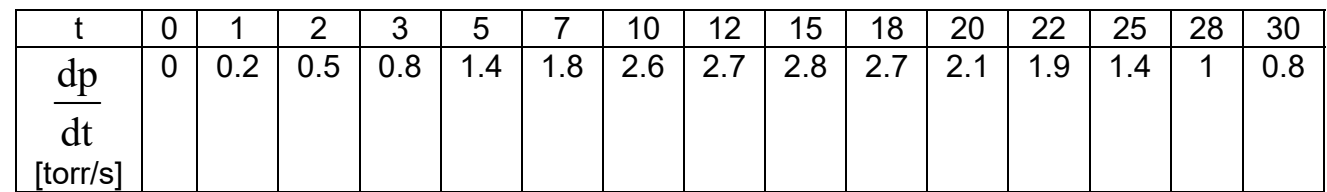

The experimental variation of the pressure in relation to time $[\mathrm{dp} / \mathrm{dt}](\mathrm{t})$ is presented, also, in Figure 4.

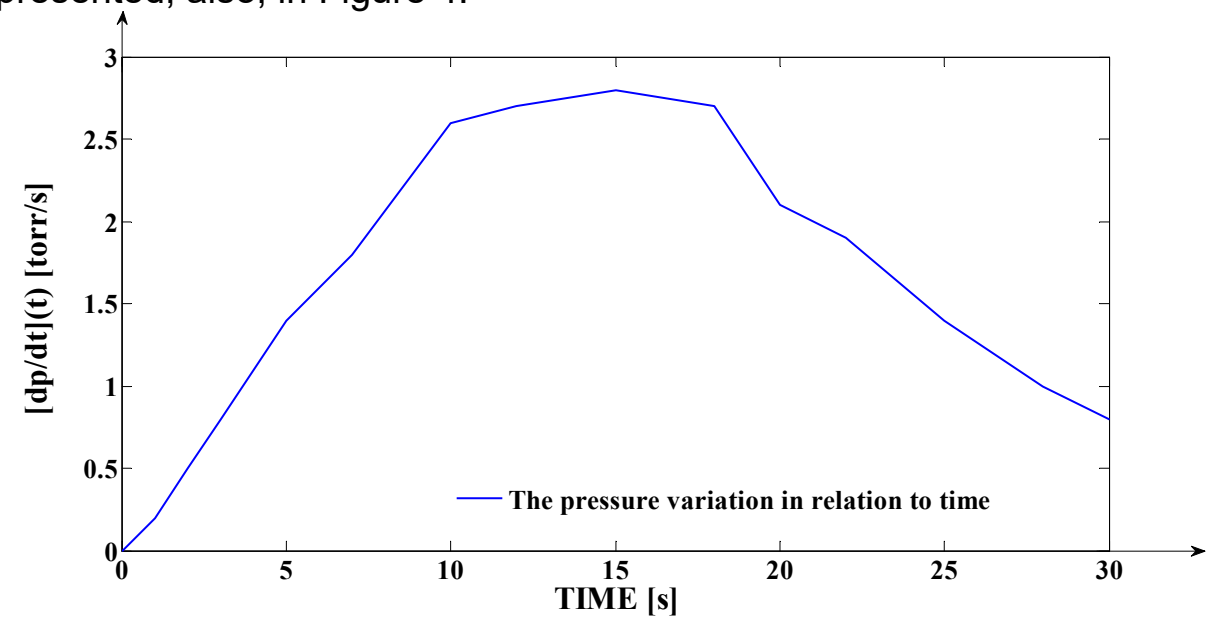

Figure 4. The $[\mathrm{dp} / \mathrm{dt}](\mathrm{t})$ function

Using the experimental data from Table 2, the pressure evolution $p(t)$ in relation to time (over the initial condition), during the experiment, can be determined according to Table 3. 
Table 3. The pressure evolution in relation to time

\begin{tabular}{|c|c|c|c|c|c|c|c|c|c|c|c|c|c|c|c|}
\hline $\mathrm{t}$ & 0 & 1 & 2 & 3 & 5 & 7 & 1 & 1 & 15 & 18 & 20 & 22 & 25 & 28 & 30 \\
\hline $\begin{array}{c}\sum \frac{\mathrm{dp}}{\mathrm{dt}} \cdot \Delta \\
\text { [torr] }\end{array}$ & 0 & 0. & 0. & 1. & 2. & 4. & 7. & 1 & 12. & 15. & 17. & 19. & 20. & 21. & 22. \\
\hline
\end{tabular}

In order to approximate the transformation of $\frac{d p}{d t}(t)$ in $p(t)$, the graphanalytical integration of the data from Table 2 is being applied, by using the following equation:

$$
p(t) \cong \sum_{i=0}^{14} \frac{d p}{d t}\left(t_{i}\right) \cdot \Delta t_{i}
$$

where $\left(\Delta t_{i}\right)$ is the graph-analytical integration step, for $i=0,1,2, \ldots 14$. Due to the fact that the curve in Figure 4 is referring to $\frac{\mathrm{dp}}{\mathrm{dt}}(\mathrm{t})$, the initial pressure (associated to the initial conditions) become null through differentiation. Knowing the initial pressure of $p_{0}=585$ torr. the real pressure is given by:

$$
p_{1}(t)=p_{0}+p(t)
$$

Consequently, $p(t)$ represents an over-pressure in relation to $p_{0}$. The $p(t)$ evolution is presented in Figure 5 (using the data from Table 3).

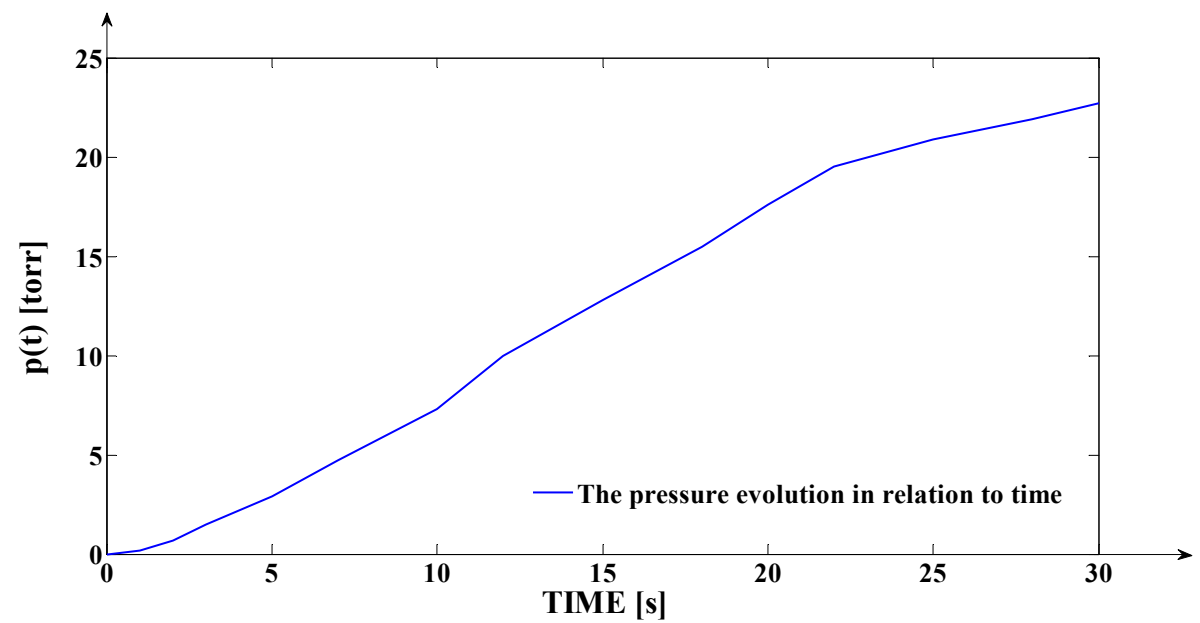

Figure 5. The $\mathrm{p}(\mathrm{t})$ evolution 
According to Figure 4, the maximum value of $[\mathrm{dp} / \mathrm{dt}](\mathrm{t})$ function is reached for $t=15 \mathrm{~s}$, aspect which implies the fact that the inflection point of the curve presented in Figure $5(p(t))$ is given by $t=15 \mathrm{~s}$, too.

The experimental data associated to the evolution of the temperature $\Delta \mathrm{T}(\mathrm{t})$, over the initial temperature of $\mathrm{T}_{0}=870 \mathrm{~K}$ and in relation to time, are presented in Table 4.

Table 4. The temperature evolution in relation to time, over $\mathrm{T}_{0}$

\begin{tabular}{|c|c|c|c|c|c|c|c|c|c|}
\hline $\mathrm{t}[\mathrm{s}]$ & 0 & 13.25 & 14.4 & 14.9 & 15.01 & 15.05 & 15.1 & 15.16 & 15.21 \\
\hline$\Delta \mathrm{T}[\mathrm{K}]$ & 0 & 100 & 200 & 300 & 400 & 500 & 600 & 700 & 800 \\
\hline
\end{tabular}

Also, considering the initial temperature, too, the temperature evolution in relation to time is presented in Table 5.

Table 5. The temperature evolution in relation to time

\begin{tabular}{|c|c|c|c|c|c|c|c|c|c|}
\hline$t[s]$ & 0 & 13.25 & 14.4 & 14.9 & 15.01 & 15.05 & 15.1 & 15.16 & 15.21 \\
\hline$T[K]$ & 870 & 970 & 1070 & 1170 & 1270 & 1370 & 1470 & 1570 & 1670 \\
\hline
\end{tabular}

Using the data from Table 5, the temperature evolution is presented in Figure 6.

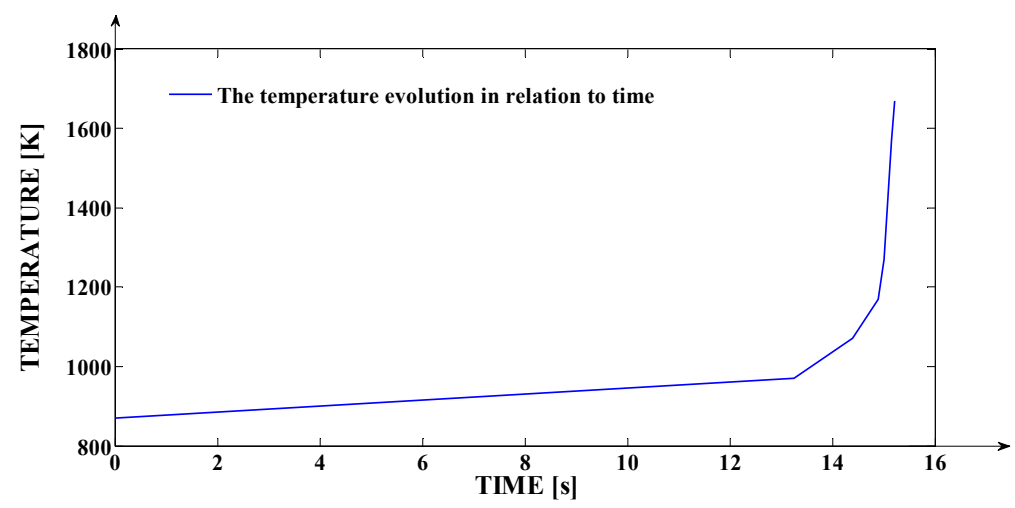

Figure 6. The $T(t)$ evolution

In Figure 6, the explosion is highlighted around the moment $t=15 \mathrm{~s}$ through the high temperature values. 
For modelling-simulation the thermal explosion of oxyhydrogen gas initiated by the wall of a quartz reactor, the proposed method is applied. The approximating analytical solutions for the $p(t)$ and $T(t)$ evolutions, are given by:

- for the $p(t)$ overpressure (in relation to the initial conditions):

$$
y_{p A N}(t)=\left(1-\frac{T_{p 1}}{T_{p 1}-T_{p 2}} \cdot e^{-\frac{t}{T_{p 1}}}-\frac{T_{p 2}}{T_{p 2}-T_{p 1}} \cdot e^{-\frac{t}{T_{p 2}}}\right) \cdot K_{p} \cdot u_{p}(t)
$$

- for the $\mathrm{T}(\mathrm{t})$ temperature:

$$
\mathrm{y}_{\mathrm{TAN}}(\mathrm{t})=\mathrm{T}_{0}+\left(\mathrm{e}^{-\frac{\mathrm{t}}{\mathrm{T}_{\mathrm{T} 1}}}+\mathrm{e}^{-\frac{\mathrm{t}-\mathrm{t}_{1}}{\mathrm{~T}_{\mathrm{T} 2}}}\right) \cdot \mathrm{u}_{\mathrm{T}}(\mathrm{t})
$$

In the first equation $T_{p 1}=8 \mathrm{~s}$ and $T_{p 2}=10 \mathrm{~s}$ are the time constants of the pressure dynamics, $K_{p}=26.8$ torr/s is the proportionality constant of the pressure dynamics and $u_{p}(t)$ is the input signal (this signal is a step unit signal which presents the commutation at the moment $t_{0}=0$, it being introduced in the model the moment of finalizing the reactor preparation for the experiment).

In the second equation $\mathrm{T}_{\mathrm{T} 1}=2.89 \mathrm{~s}$ and $\mathrm{T}_{\mathrm{T} 2}=0.298 \mathrm{~s}$ are the time constants of the temperature dynamics, $T_{0}=870 \mathrm{~K}$ is the initial temperature, $\mathrm{u}_{\mathrm{T}}(\mathrm{t})$ is the input signal having a similar form and modelling the same action as $u_{p}(t)$, respectively $t_{1}=13.3 \mathrm{~s}$ is a delay constant.

The mentioned structure parameters (the mentioned constants) values are identified using iterative identification algorithms based on processing the experimental data.

The comparative graph between the experimental $p(t)$ curve and the simulated one (obtained by applying the proposed simulation method) is presented in Figure 7.

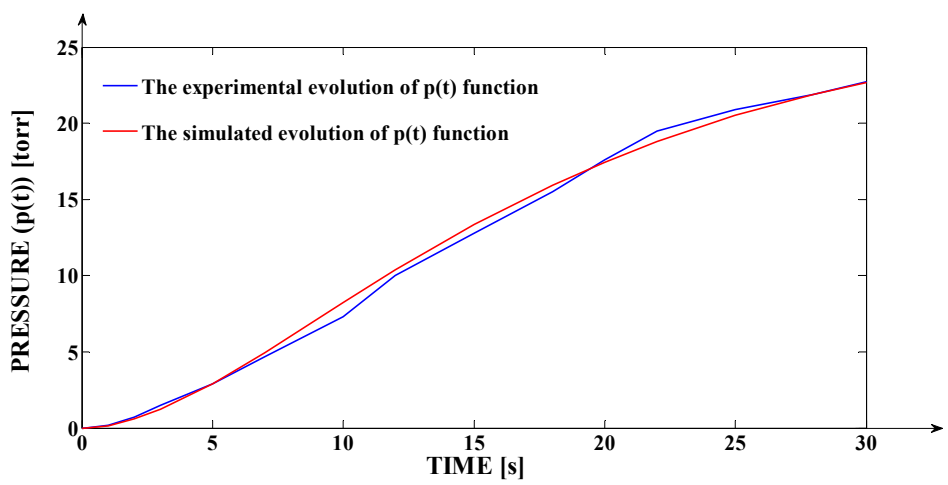

Figure 7. The comparative graph between the experimental $p(t)$ evolution and the simulated one 
From Figure 7, the high efficiency of the proposed modelling-simulation method results through the good superposition of the simulated curve over the experimental one. The high precision is, also, proven through the insignificant value of the cumulated error in percentage computed for the simulated curve in relation to the experimental one: $\mathrm{CREP}_{\mathrm{P}}=0.4903 \%$ (error which in absolute values is proportional with $10^{-3}\left(4.903 \cdot 10^{-3}\right)$.

Also, based on using the proposed model for the $p(t)$ dynamics in relation to time, the pressure variation $[\mathrm{dp} / \mathrm{dt}](\mathrm{t})$ can be simulated. Hence, the comparative graph between the experimental pressure variation $([\mathrm{dp} / \mathrm{dt}](\mathrm{t}))$ curve and the simulated one (obtained by applying the proposed simulation method) is presented in Figure 8.

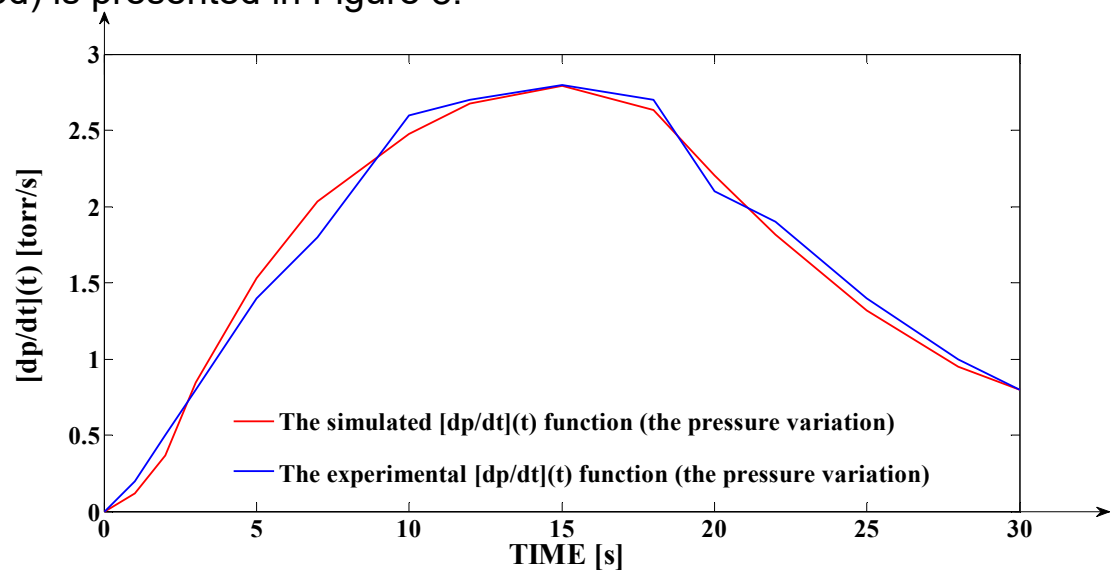

Figure 8. The comparative graph between the experimental pressure variation $[\mathrm{dp} / \mathrm{dt}](\mathrm{t})$ evolution and the simulated one

The same conclusions as in the case of the comparison presented in Figure 7 result. The conclusions are proven through the insignificant value of the cumulated error in percentage computed for the simulated curve in relation to the experimental one: $\mathrm{CREP}_{\mathrm{DP}}=0.6408 \%$ (error which in absolute values is proportional with $10^{-3}\left(6.408 \cdot 10^{-3}\right)$.

The comparative graph between the experimental $\mathrm{T}(\mathrm{t})$ curve and the simulated one (obtained by applying the proposed simulation method) is presented in Figure 9. As in the previous two simulations, the same conclusions result. In this case, too, the value of the cumulated error in percentage computed for the simulated curve in relation to the experimental one is an insignificant one: $\mathrm{CREP}_{\mathrm{T}}=1.8478 \%$ (error which in absolute values is proportional with $10^{-2}\left(1.8478 \cdot 10^{-3}\right)$. The sensible higher value of $\mathrm{CREP}_{\mathrm{T}}$ than $\mathrm{CREP}_{\mathrm{P}}$ and $\mathrm{CREP}_{\mathrm{DP}}$ is due to the consistent higher absolute values of the temperature $T(t)$ than the values of the other signals $(p(t)$ and 
[dp/dt](t)). Practically, by descending scaling of the CREP error with 100 (the approximate proportion between the mentioned values), in the case of the temperature modelling-simulation we have obtained even better results than in the cases of pressure, respectively of pressure variation.

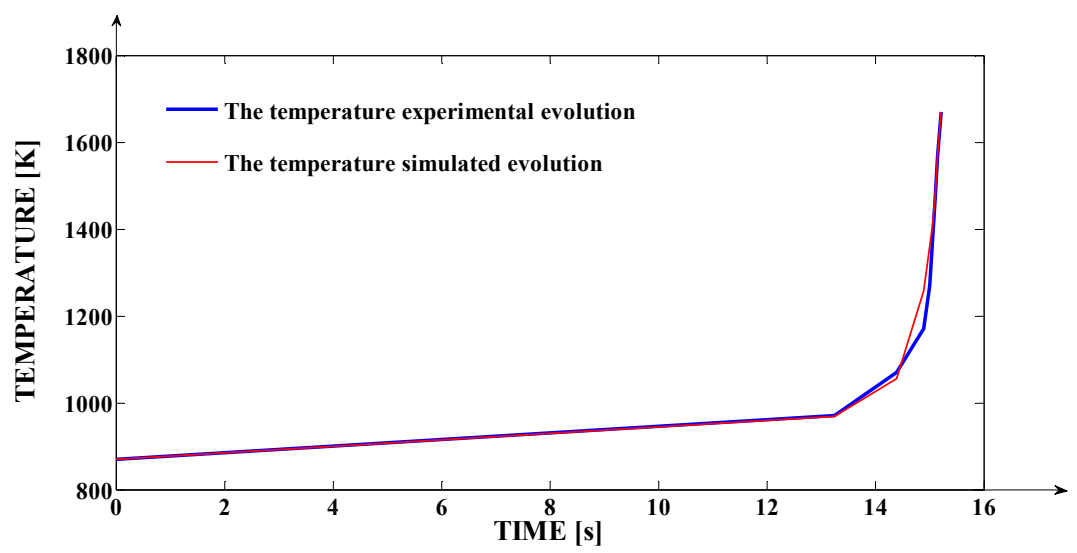

Figure 9. The comparative graph between the experimental temperature evolution and the simulated one

\section{CONCLUSIONS}

For certain cases, mathematical modeling is the only available predictive tool, and for other cases, mathematical modeling is one of the effective approaches. The usefulness of the results depends firstly on accuracy of mathematical model.

The explosions are non-linear phenomena and the modeling procedures which can be applied for obtaining their mathematical model are complex ones. Also, the identification methods used for determining the structure parameters of non-linear processes are laborious due to the fact that they are complex extensions of the identification methods which can be applied in the case of linear processes. Considering these aspects, in many cases, when other possibilities are available, the direct modelling of nonlinear processes using non-linear models should be avoided. In this context, we have proposed a solution to solve and simplify the modelling of explosions by "avoiding of using a complex non-linear model. This solution is based on dividing the time period associated to the explosion in two intervals, the first interval corresponding to the increasing stage of the explosion (as intensity) and the second one corresponding to the decreasing 
one. Practically, both stages can be modelled separately as linear processes, but the entire explosion phenomenon remains non-linear. More exactly, the explosion model is non-linear on the entire time period but is linear on intervals and we model separately each interval. In this paper, as study case, only the increasing stage of the approached explosions are modelled, but the decreasing stage modelling is similar as applied procedures. However, the proposed algorithm can be modified in order to have the possibility to apply it in order to model-simulate the explosion as a unitary non-linear phenomenon, but the associated computations are more complex: (1) has to be modified at the form $x=f(x, u)$ and (2) has to be modified at the form $y=g(x, u)$, where both $f$ and $g$ functions are non-linear).

For the studied process, the thermal explosion, it can be concluded that the algorithm presented by this research contributes to a high interpretation of the extremely short period of the explosion phenomena, analyzed from the perspective of pressure, temperature and concentration evolution in time, both in steady and transient states. The accuracy of the model is proved by the very small values of the relative error cumulated in percentages.

\section{REFERENCES}

1. V. Novozhilov; Sci Rep, 2016, 6, 29730.

2. A.N. Campbell; Phys Chem Chem Phys, 2015, 17(26), 16894-906.

3. V. Novozhilov; Sci Rep, 2018, 8(1), 4030.

4. A.K. Oppenheim; Chemical Kinetic Aspects, Dynamics of Combustion Systems, 2 nd ed.; Springer Berlin Heidelberg Publisher, 2008, 81-113.

5. E. Glascoe; H. Keo Springer; J.W. Tringe; L. Maienschein; Bull Am Phys Soc, 2011, 56 (6), 347-351.

6. C.Y. Chan; P.C. Kong; Appl Math Comput, 1995, 71(2-3), 201-210.

7. G.N. Gorelov; V.A Sobolev; Combust Flame, 1991, 87 (2), 203-210.

8. K. Takashi; Critical Temperatures for the Thermal Explosion of Chemicals, Hardbound, 2005, 406.

9. N.N. Semenov; AN SSSR, 1959.

10. D.A. Frank-Kamenetsky; Diffusion and Heat Transfer in Chemical Kinetics, Moscow, Nauka, 1967.

11. O.M. Todes; P.V. Melent'ev; J Phys Chem,1939, 13 (7), 52-58.

12. A.G. Merzhanov; F.I. Dubovitsky; UChN, 1966, 35(4), 656-683.

13. B.F. Gray; Combust Flame, 1973, 21, 317-325.

14. R. Almeida; N.R.O. Bastos; M.T.T. Monteiro; Math. Methods Appl. Sci., 2016, 39(16), 4846-4855. 
15. B.E. Zhestkov; S.N. Kozlov; E.N. Alexandrov; High Temp, 2019, 57(3), 329334.

16. X. Hu; Q. Xie; J. Zhang; Q. Yu; H. Liu; Yasong Sun; Int. J. Hydrog. Energy, 2020, 45(51), 7837-27845.

17. K. Allali; Y. Joundy; A. Taik; Mathematical Modelling of Natural Phenomena, 2019, 14, 602-614.

18. E.N. Aleksandrov, N.M. Kuznetsov, S.N. Kozlov; Combust. Explos. Shock Waves, 2010, 46, 533-540.

19. T. Coloşi; M. Abrudean; M.-L. Ungureşan; V. Mureşan; Numerical Simulation Method for Distributed Parameters Processes using the Matrix with Partial Derivatives of the State Vector, Ed. Springer, 2013, pg. 343. 